Meta

Journal des traducteurs

Translators' Journal

\title{
Le rôle de la traduction dans la défense et l'illustration de la langue française au Québec
}

\section{Jean-Claude Gémar}

Volume 31, numéro 4, décembre 1986

URI : https://id.erudit.org/iderudit/002857ar

DOI : https://doi.org/10.7202/002857ar

Aller au sommaire du numéro

Éditeur(s)

Les Presses de l'Université de Montréal

ISSN

0026-0452 (imprimé)

1492-1421 (numérique)

Découvrir la revue

Citer cette note

Gémar, J.-C. (1986). Le rôle de la traduction dans la défense et l'illustration de la langue française au Québec. Meta, 31(4), 458-460.

https://doi.org/10.7202/002857ar d'utilisation que vous pouvez consulter en ligne. 


\section{LE RÔLE DE LA TRADUCTION DANS LA DÉFENSE ET L'ILLUSTRATION DE LA LANGUE FRANÇAISE AU QUÉBEC}

On sous-estime parfois le rôle joué par la traduction dans le développement de la pensée et de la vie culturelle, en particulier lorsque l'activité traduisante passe pour un art littéraire mineur. Si la place de la traduction dans les domaines intellectuel, artistique, scientifique, etc., est indéniable, ses fonctions linguistiques, et plus particulièrement sociolinguistiques, sont moins connues parce que leur étude passe par un certain nombre de disciplines, dont la psychologie, la sociologie, l'histoire, la théorie de la communication, etc., qui font appel à des données plus "scientifiques" que littéraires. Parmi ces fonctions, i en est une dont les incidences présentent un grand intérêt pour l'observateur du langage parce qu'elle peut être amenée à jouer un rôle capital dans un pays où, pour des raisons historiques et géopolitiques, la traduction est devenue le principal véhicule de l'information dans les domaines politique, commercial, financier, scientifique et technique. Cette fonction de type supérieur, c'est la fonction exemplaire, pédagogique, que peut assumer la traduction lorsqu'elle se transforme en instrument d'épuration, d'enrichissement et, stade suprême, d'illustration d'une langue, le français en l'occurrence, dans un contexte bilingue de fait sinon de droit comme celui du Québec.

Nous allons voir comment une langue vernaculaire, placée sous la tutelle d'une langue de contact véhiculant un modèle social prestigieux, a été emportée au fond de l'abîme linguistique par la vague d'un bilinguisme irrésistible et comment les traducteurs sont non seulement parvenus à lui faire remonter la pente, à revaloriser son statut par opposition à la langue dominante, l'anglais, mais encore à susciter une renaissance - la "francisation" - et à lui insuffler une vigueur, une fraîcheur remarquables.

\section{TRADUCTION ET CONTEXTE SOCIOPOLITIQUE}

La réception d'une langue étrangère dans une communauté linguistique donnée dépend de plusieurs facteurs, notamment de celui que représente le prestige de cette langue aux yeux du groupe récepteur, pour nombre de raisons - politiques, économiques, scientifiques, littéraires, artistiques... -, de même que du contexte dans lequel s'effectue son entrée: guerre ou paix. Selon le cas, il peut y avoir soit rejet partiel ou total pour cause d'hostilité envers la langue d'un ennemi, d'un intrus, soit, tout au contraire, assimilation plus ou moins profonde d'une grande langue de civilisation auréolée d'une histoire, d'une culture et d'une œuvre intellectuelle particulièrement riches, tels le grec et le latin en Europe, par exemple. Les sources des langues romanes sont connues et l'on sait la place qu'occupait déjà l'écrit à Rome et dans l'Empire romain par rapport à la tradition orale chez les " barbares"; de là viendrait en partie le prestige parfois excessif auréolant la langue écrite que l'on constate chez la plupart des francophones. Sinon, comment expliquer le phénomène que représente l'assimilation totale et sans heurts notables du français parlé en Normandie au XI ${ }^{\mathrm{e}}$ siècle, après une longue gestation de plusieurs siècles, par ceux-là mêmes qui furent vaincus à Hastings en 1066 ? C'est le contraire qui eût dû se produire, soit l'assimilation des idiomes locaux par la langue des vainqueurs, de la même façon que la langue d'oill finit par s'imposer à la langue d'oc en France.

Plus près de nous, au Canada, quelque trois siècles de domination jalonnés de confrontations, de décisions judiciaires et de mesures administratives souvent arbitraires, parfois accompagnées de violence comme la déportation des Acadiens en 1755 , n'ont pu venir à bout de la langue françaisc. Îlot fragile et menacé dans l'immense océan anglophone, le français, langue de la minorité, a survécu. L'ironie de l'Histoire a voulu que la défense des deux grandes langues de culture et de civilisation que sont l'anglais et le français, en Angleterre comme au Québec - et même en France! - ait été confiée au Parlement, lequel dut recourir à la loi pour imposer l'usage d'une langue nationale ou officielle ${ }^{1}$. Dans le cas du Québec, les lois votées en matière linguistique entre 1969 et 1977 sont le recours ultime d'un peuple contre le danger que représente l'influence linguistique étrangère et constituent le plus récent épisode de la lutte séculaire que livre un peuple pour préserver une identité culturelle que symbolise l'usage libre et entier de sa langue, le français.

Le rôle de la traduction, dans ces conditions, sera très différent selon le contexte dans lequel elle est pratiquée: unilingue - en France, par exemple -, bilingue ou multilingue.

En situation d'unilinguisme, l'impact de la traduction, pour ce qui est des principales langues véhiculaires, est plutôt faible parce que ces langues ont atteint un stade avancé de maturité, de stabilité et d'autonomie, à partir duquel le besoin de recourir à la traduction comme instrument d'enrichissement n'est pas ressenti comme essentiel. Dans les pays où ces langues sont utilisées la traduction peut quelquefois être tenue pour une activité secondaire, un facteur négligeable de consolidation du patrimoine linguistique, au point que l'on n'hésitera pas à recourir fréquemment à l'emprunt lexical ${ }^{2}$.

Dans les pays bilingues ou multilingues, on constate généralement une tout autre attitude. I convient toutefois d'ajouter que les conditions dans lesquelles est né et s'est développé le bilinguisme ou le multilinguisme - des habitants d'un même pays déterminent cette attitude. Lorsqu'un groupe linguistique donné a imposé une situation de bilinguisme par la force, la violence ou plus simplement par le caractère de "supériorité» qui reste attaché à une langue - coloniale, par exemple - par rapport à un ou à des idiomes locaux, infériorisćs d'emblée, la traduction jouera à sens unique en faveur de la langue de civilisation et sera en conséquence mal acceptée, et peut-être rejetée. Par ailleurs, si deux langues de civilisation sont en contact étroit, la survie du 
groupe numériquement ou économiquement faible pourra dépendre d'une activité de traduction bien organisée afin d'éviter l'asphyxie qui menace une langue trop longtemps coupée de ses sources. Dans les deux cas, la traduction ne sera qu'un acte de communication unilatérale et non une ressource intellectuelle, esthétique ou artistique, aussi la qualité de la langue d'arrivée s'en ressentira-t-elle.

Dans le cas singulier du Québec, la langue française a connu bien des heurs et malheurs avant d'atteindre son stade actuel de redressement, toujours fragile et menacé. Son évolution s'est déroulée en trois grandes étapes dont la traduction constitue le dénominateur commun, et les traducteurs les acteurs principaux.

\section{LES TROIS ÉTATS DE LA LANGUE AU QUÉBEC}

En gros, la première période commence avec l'histoire de la Nouvelle-France et se termine en 1759, date de la reddition de Québec à partir de laquelle vont se développer les conditions du bilinguisme qui conduira à une rapide détérioration de la qualité du français parlé et surtout écrit au BasCanada. Durant cette période, la langue française n'est pas encore coupée de ses racines et se perpétue avec la vigueur et le dynamisme légendaires des pionniers de l'époque. Après la conquête, FrançoisJoseph Cugnet est le premier - et le seul - mainteneur d'un niveau minimal de qualité linguistique qu'il sut préserver durant les années où il remplit les fonctions de traducteur officiel, le premier de l'histoire du Canada ${ }^{3}$. À la suite des capitulations de Québec et de Montréal, l'avènement du bilinguisme devait toutefois battre en brèche les efforts de Cugnet dès avant la fin du siècle. 11 convient de rappeler que les textes des capitulations ne prévoyaient aucune disposition relative à la "protection de la langue française" ${ }^{4}$, ce qui ne pouvait qu'aggraver la situation.

Avec la conquête commence, selon Pierre Daviault, la "période noire" qui devait durer près d'un siècle, soit jusqu'à la Confédération (1867), pour fixer une limite approximative. La langue anglaise gagne en influence sur le français, les premiers journaux bilingues font leur apparition ainsi que les traducteurs, qui, dans le rapide processus de détérioration de la langue française qui s'ensuivit, devaient tenir un rôle qu'ils éprouvent encore aujourd'hui le plus grand mal à faire oublier 5 . Dans tous les domaines, le constat de décadence de la langue des Canadiens français contaminée par l'anglais est accablant ${ }^{6}$. Les méfaits de la traduction, au Québec comme sur l'ensemble du territoire canadien, sont la cause d'un phénomène d'acculturation plus ou moins prononcée selon les groupes considérés?

Depuis lors, la longue traversée du désert que les traducteurs ont entreprise pour arracher la langue française à la fondrière où elle s'était enfoncée, en partie par leur faute, semble approcher du but qu'ils se sont fixé, après un séjour de plus de deux siè- cles au purgatoire au cours duquel les Canadiens français ont vu s'ouvrir devant eux une carrière d'avenir, celle de traducteur, alors que depuis la conquête nombre de portes leur avaient été fermées.

\section{LA RENAISSANCE DE LA LANGUE FRANÇAISE}

Le redressement de la langue française s'est déroulé sur une longue période, de façon très lente, peu spectaculaire, grâce en partie au travail discret mais efficace des traducteurs. La pression des institutions britanniques et de la langue anglaise se fit particulièrement sentir entre 1790 et 1855 , année où la venue de la Capricieuse au Canada donna une impulsion décisive à la langue française, qu'elle remit en honneur. Par la suite, l'action éclairée d'Antoine Gérin-Lajoie entraîna la réorganisation des bureaux de traduction de l'Assemblée législative, contribuant ainsi puissamment à l'amélioration constante des traductions. Avec la Confédération (1867), une nouvelle étape est franchie: la traduction devient un service public. La période moderne toutefois ne débute véritablement qu'en 1934, année décisive où est créé le Bureau des traductions. En 1969, la loi sur les langues officielles confirme le statut d'égalité des deux langues officielles du Canada et marque le commencement de la période de renaissance du français, la "refrancisation". Dans les années soixante, en effet, la langue française rebondit sous la poussée de la "révolution tranquille" du Québec et entame son processus d'indépendance linguistique étayé par un puissant courant nationaliste qui, libéré de la tutelle d'un bilinguisme évocateur d'un rapport historique de dominant à dominé, reporte ses frustrations et ses espérances sur la langue érigée en symbole de nationalisme populaire et du " pouvoir fondateur du langage" dont parle Émile Benvéniste.

Au cours des vingt dernières années, l'activité traduisante a connu une accélération étonnante dans le monde, mais nulle part ailleurs qu'au Québec celle-ci s'est manifestée avec autant de force et d'ampleur et a contribué avec plus d'efficacité au redressement d'une langue, au point que Vinay a pu dire des traducteurs canadiens que leur "rôle dépasse [...] de très loin celui du traducteur européen ou américain [...] au Canada, ils sont responsables de l'évolution de la langue» 8 . On peut ajouter à cette remarque que la responsabilité du traducteur est très lourde, particulièrement dans le contexte de bilinguisme anglaisfrançais et des problèmes spécifiques qu'il pose, puisqu'il doit faire auvre tour à tour de philologue, grammairien, terminologue, rédacteur, etc., outre les connaissances indispensables du domaine dans lequel il traduit, afin d'exprimer les idées contenues dans la langue de départ de manière conforme aux servitudes de la langue d'arrivée, tout en respectant les impératifs de la communication. Le volume des textes traduits au Canada, surtout au Québec et en Ontario, ne cesse de croître, dans le secteur public comme dans le secteur privé. Ces traductions, on les doit non plus à quelques grands seigneurs comme 
l'étaient les traducteurs des $\mathrm{XVIII}^{\mathrm{e}}$ et $\mathrm{XIX}^{\mathrm{e}}$ siècles, mais à une armée de traducteurs professionnels rcgroupés au sein d'associations solidement structurées et bien organisées ${ }^{9}$. Si le rôle de la traduction dans l'évolution d'une langue n'est guère contestable, il semble bien qu'au Québec la traduction a non seulement favorisé l'enrichissement de la langue française, mais encore a contribué à la libérer progressivement et sans éclats de l'étau où l'avait enserrée l'anglais.

Un dernier élément de la francisation, dont il faut tenir compte, est l'action interventionniste et capitalc que l'État, véritable planificateur linguistique, a choisi de mener depuis une vingtaine d'années, action dont le point culminant est la Charte de la langue française - communément appelée loj 101 adoptée par l'Assemblée nationale du Québec en 1977. De caractère essentiellement privé, puis parapublic depuis quelque deux cents ans, la défense de la langue française, qui était l'apanage des traducteurs, est devenue une affaire d'État ${ }^{10}$, et même d'États l'État fédéral disputant ce privilège au Québec puisque l'intérêt de tout un peuple, la survie de son identité culturelle, est en jeu. Cette évolution est conforme à la logique et à l'ordre des choses: ayant accompli leur mission historique, atteint une grande partie de leurs objectifs, les traducteurs peuvent passer le témoin aux pouvoirs publies, qui, de nos jours, appuient de plus en plus souvent l'initiative privée, quand ils ne la remplacent pas purement et simplement. Toutefois, cet effacement ne signifie pas abandon de leurs responsabilités car ils continuent tout aussi efficacement et discrètement qu'auparavant à exercer leur rôle de surveillance, de maintien et de renforcement de la qualité de la langue qu'ils ont pour mission de servir.

\section{CONCLUSION}

Peu à peu, partant d'une situation où la traduction occupait une position centrale dominante dans l'activité politique, économique et culturelle du pays, conditions faisant de la langue francaise un moyen d'expression servile d'une pensée et d'une culture étrangères, par réaction d'orgueil nationaliste et par le truchement d'une volonté politique bien arrêtée de jouer à fond le jeu de la francisation, le Québec a pu surmonter les terribles handicaps que l'Histoire lui avaient imposés, s'affranchir de la tutelle coloniale instaurée par une des grandes langues de notre civilisation et recréer les conditions linguistiques propices à l'épanouissement d'une culture originale. La langue française du Québec y a gagné en fraîcheur et en dynamisme, ce qui n'est pas sans rappeler par certains côtés l'âge de la Renaissance, la Pléiade et Rabelais. Cette renaissance d'une langue, n'est-ce pas là une des réussites les plus réjouissantes de la traduction, une de ses fonctions les plus nobles, celle en qui Heidegger voyait " une bénédiction pour les peuples"?

JEAN-CLAUDE GÉMAR

Université de Montréal

Notes et renvois

1. À trois teprises, en 1362,1650 et 1731 , le Parlement britannique légiféra pour imposer l'usage de l'anglais comme langue juridique et gifera pour in bannir celuj du Law French, dernier retranchement notable de la languc française, qui n'était plus, au XVIII ${ }^{\mathrm{e}}$ siècle, qu'un jargon profesBaker (1979): Manual of Law French, Amersham (England), Avery, Baker (1979): Manual of Law French, Amersham (England), Avery, particulièrement aux pages 9-14. Au Québec, l'Assemblée nationale a
légiféré trois fois (en 1969, 1974 et 1977) pour imposer le français légiféré trois fois (en 1969, 1974 et 1977) pour imposer le français
comme seule "langue officielle du Québec" (Charte de la langue française, dite loi 101). En France, l'État a également recouru à la loi pour tenter d'imposer l'emploi de la langue française jugée menacée, à divers moments de son histoire: au XVI ${ }^{\mathrm{e}}$ siècle, pendant la Révolution, et en 1975 (loi du 31 décembre 1975, dite loi Bas-Lauriol, du nom de ses auteurs).

2. L'exemple des pays du Marché commun est édifiant à cet égard. La presse, la radio et la télévision puisent abondamment dans la langue anglaise de nombreux termes dont elles émaillent des propos qui, sans cela, seraient jugés sans intérêt, démodés et sans saveur par un public conditionné et avide d'un certain exotisme linguistique primaire. $\mathrm{Ce}$ suivisme à l'égard de la langue anglaise ne traduit pas seulement une mode teinte de mode teintée de snobisme, il révèle le prestige et la puissance d'attraction d'une langue qui incarne un type de culture et de développernent prestigieux. Cette attitude n'est pas dénuée d'ambiguïté: d'un côté, on ne se prive pas de critiquer le modèle américain et ses excès; de l'autre, l'on est fasciné par la langue et la culture qu'il véhicule, au point de parler et d'écrire - en France, par exemple - ce qu'Étiemble a baptisé "franglais".

3. Pour plus de détails sur Cugnet et la traduction à son époque, voir lérude de Paul A. Horguelin (1977): "Les premiers traducteurs", Meta, vol. 22, n 1, mars, numéro spécial sur l'Histoire de la traduction Meta, vol. 22, $\mathrm{n}^{\circ} 1$, mars,
au Canada, p. 15 et suiv.

4. Selon l'observation de Benjamin Sulte (1898): in la Langue française au Canada, Lévis, P.-G. Roy.

5. Ce sujet est traité de façon plus détaillée par Paul A. Horguelin, supra; voir aussi: Jacques Gouin: "La traduction au Canada de 1791 à pra; voir aussi: Jacques Gouin: "La traduction au Canada de 1791 à et traduction au Canada", in Mémoires de la Société royale du Canada, tome 34 .

6. Cr. Jacques Gouin, supra. Voir aussi Louis Beaudoin (1967): les Aspects généraux du droit privé dans la province de Ouébec, Paris, Dalloz, plus particulièrement le chap. V, p. 37 et suiv.; André Morel (1978): "La réception du droit criminel anglais au Québec (1760-1892)", 13 Revue juridique Thémis, pp. 449-541. Ces deux derniers auteurs traitent abondamment de l'impact de la langue (juridique) anglaise dans deux très importants secteurs de l'activité sociale, le droit privé et le droit pénal (la Justice)

7. Cf. Jacques Poisson (1978): " La traduction, facteur d'acculturation?", in la Traduction, une profession (Actes du VIII' congrès mondial de la Fédération internationale des traducteurs, Montréal, 1977), Conseil des traducteurs et interprètes du Canada, pp. 281-291; Jean Delisle: " Projet d'histoire de la traduction et de l'interprétation au Ca-
nada", Meta, op. cit., p. 69 .

8. Extrait de "La traduction, une profession", in la Traduction, une profession, op. cit., p. 22

9. Au Québec, par exemple, la Société des traducteurs du Québec regroupe quelque 1500 membres.

10. Historiquement, à partir de 1934 (création du Bureau des traductions), mais en pratique c'est ayec la révolution tranquille que s'est engagé le mouvement d'intervention étatique dans le domaine linguistique. 